\title{
Gamma Rays from Super-Heavy Relic Particles in the Halo
}

\author{
Pasquale Blasi \\ Department of Astronomy \& Astrophysics, and \\ Enrico Fermi Institute, The University of Chicago, \\ 5640 South Ellis Avenue, Chicago, IL 60637
}

\begin{abstract}
Superheavy (SH) quasistable particles generated in the Early Universe could be responsable for Ultra High Energy Cosmic Rays (UHECR) and be a component of Cold Dark Matter (CDM) in the universe. These particles are likely to cluster in the galactic halo, so that the main part of UHECR are gamma rays produced in the decay of neutral pions. Charged pions are also produced in the same decay and result in high energy electrons. We consider here the production of gamma rays by synchrotron emission of these electrons in the galactic magnetic field. The gamma ray fluxes are above the detectability level of current and proposed experiments (e.g. EGRET, GLAST, STACEE and WHIPPLE) in the energy range $0.1-10^{4} \mathrm{GeV}$ and are compatible with the existing upper limits at $10^{5}-10^{8} \mathrm{GeV}$. The detection of this flux of gamma rays would be of key relevance to determine the importance of $\mathrm{SH}$ relic particles as sources of UHECR and give a clue on the physics of the Early Universe.
\end{abstract}

Typeset using REVTEX 


\section{INTRODUCTION}

The detection of cosmic rays well above $10^{20} \mathrm{eV}$ poses a serious challenge to our understanding of the acceleration processes as well as of the propagation of these particles from the sources. Particles with $E \geq 10^{19} \mathrm{eV}$ are thought to be of extragalactic origin since the galactic magnetic field is unable to confine and isotropize them. However if the sources are cosmological then the UHECR spectrum would have as a unique signature the presence of a pronounced cutoff (the so called GZK cutoff [2]) at $E \sim 5 \cdot 10^{19} \mathrm{eV}$, which is not observed (see [1] for a recent discussion). This limits the distance to the sources of UHECR to be less than $\sim 50 \mathrm{Mpc}$ and no plausible source has been identified by current experiments within this distance, unless very large deflection angles are assumed, which looks incompatible with the current limits on the intergalactic magnetic field (see [3] for a review and [4] for revised limits in a inhomogeneous universe).

The lack of counterparts to the observed events, together with the difficulty of the known acceleration mechanisms to reach the observed energies, prompted a new class of models (top-down models) (for a recent review see [5] and references therein) where UHECR are produced by the decay of grand unification massive bosons (generically named X-particles) with mass $m_{X} \sim 10^{13}-10^{16} \mathrm{GeV}$. These X-particles can either be produced in processes involving topological defects (TD) or by the decay of SH quasistable (lifetime $\tau$ much larger than the age of the Universe $t_{0}$ ) particles relics of the early universe [6 [10] in the assumption that they represent some fraction of the CDM.

In the latter case, the UHECR spectrum is dominated by the halo component and hence it has no GZK cutoff. Signatures of this model have been discussed in [12], where it was pointed out that if UHECR above $\sim 10^{19} \mathrm{eV}$ are produced in the halo by the decay of Xparticles, then the flux should be dominated by gamma rays produced by the decay of neutral pions produced in the hadronic cascade initiated by ultra high energy partons $(X \rightarrow q \bar{q}$, $q \rightarrow$ hadrons). The model is also characterized by some degree of anisotropy due to the asymmetric position of the sun in the galaxy [11] 13]. 
Together with neutral pions charged pions are also generated which in turn decay into electrons and positrons (hereafter we shall call them both electrons) and neutrinos.

In this paper we calculate the flux of gamma rays which is produced by synchrotron emission of the electrons in the galactic magnetic field when the density of SH particles in the halo is normalized to fit the observed UHECR flux. Our calculations show that the predicted gamma ray emission is compatible with the upper limits imposed by experiments like HEGRA, EASTOP, CASA-MIA and Utah-Michigan at $10^{5}-10^{8} \mathrm{GeV}$ and, in the energy range $0.1-10^{4} \mathrm{GeV}$ might be detectable by current or proposed experiments.

The paper is planned as follows: in section $\amalg$ we present a benchmark calculation where we estimate the magnitude of the gamma ray flux from ultra high energy electrons (UHEE) in the galactic magnetic field. In section [II] we introduce the fragmentation functions both for ordinary QCD and in the supersymmetric generalization proposed in [14]. Our conclusions are presented in section $\amalg$.

\section{AN ESTIMATE OF THE EFFECT}

The SH particles we are interested in are quasistable, which means that their lifetime $\tau_{X}$ is much larger than the age $t_{0}$ of the Universe. If $n_{0}$ is the average density of $\mathrm{SH}$ particles with mass $m_{X}$ in the halo, the decay rate can be estimated as $\dot{n}_{X}^{\text {halo }} \approx n_{0} / \tau_{X}$. The decay of an X-particle typically results in the production of a quark-antiquark pair

$$
X \rightarrow q \bar{q}
$$

and each quark produces a jet of hadrons (mainly Nucleons $(N)$ and pions $(\pi)$ ) with total energy in the jet $E_{j e t}=m_{X} / 2$. The hadron spectrum is given by the fragmentation function $W_{i}\left(x, m_{X}\right)$ where $i=N, \pi$ and $x=E_{i} / E_{j e t}=2 E_{i} / m_{X}$. Many discussions can be found in the recent literature about approximations to the fragmentation function and extrapolations of the low energy phenomenological expressions to the extremely large center of mass energy

involved in the production of UHECR by the decay of X-particles (for a recent review see 
[5]; a numerical approach involving the use of a QCD event generator was used in [7]). We address this problem in the next section, while here we shall use a very simple approximation proposed in 15, 16 and valid for $x \ll 1$. In this approach the spectrum of the pions, which represent most of the content of the jet, is given by

$$
\frac{d N_{\pi}}{d x}=\frac{15}{16} x^{-3 / 2}(1-x)^{2} \approx \frac{15}{16} x^{-3 / 2} \quad x \ll 1
$$

The spectrum of the nucleons has the same form as in eq. (11) but the normalization is such that the ratio $N / \pi$ in the jet is $\sim 0.05$ as suggested by the decay $Z_{0} \rightarrow$ hadrons at LEP. Moreover each pion family will take approximately $1 / 3$ of the total pion content in the jet.

The small $N / \pi$ ratio at the production implies that on galactic distance scales the flux of UHECR produced by the decay of X-particles is dominated by the gamma rays produced in the decay of neutral pions. Therefore, in the following we shall estimate the rate $\dot{n}_{X}^{\text {halo }}$ of decay, requiring that the flux of gamma rays equals the flux of UHECR at $10^{19} \mathrm{eV}$. For the sake of simplicity we shall also assume here that the SH particles are distributed homogeneously in the halo whose size is $R_{H}$. The flux of gamma rays with energy $E_{\gamma}$ at the Earth is then easily derived to be

$$
I_{\gamma}^{U H E}\left(E_{U H E}\right)=2 \times \frac{1}{3} \dot{n}_{X}^{\text {halo }} \frac{R_{H}}{4 \pi} \int_{E_{U H E}}^{E_{\text {jet }}} d E_{\pi} \frac{d N_{\pi}}{d E_{\pi}} \frac{2}{E_{\pi}}=\frac{5}{6} \dot{n}_{X}^{\text {halo }} \frac{R_{H}}{4 \pi}\left(\frac{m_{X}}{2}\right)^{1 / 2} E_{U H E}^{-3 / 2},
$$

where the factor 2 in front takes into account the two jets and the factor $1 / 3$ accounts for the $\pi^{0}$ 's only. Moreover we used $d N_{\pi} / d E_{\pi}=\left(2 / m_{X}\right) d N_{\pi} / d x$.

The comparison of our prediction with the observed flux of UHECR at $E=10^{10} \mathrm{GeV}$ immediately gives

$$
\dot{n}_{X}^{\text {halo }} \approx 1.8 \cdot 10^{-42}\left(\frac{m_{X}}{10^{14} \mathrm{GeV}}\right)^{-1 / 2}\left(\frac{R_{H}}{100 k p c}\right) \mathrm{cm}^{-3} \mathrm{~s}^{-1} .
$$

A fraction $2 / 3$ of the energy in the jet is transformed into charged pions which contribute an electron flux through production and decay of muons. The density spectrum of these electrons can be calculated in the standard way (muon decay) and gives

$$
q_{e}\left(E_{e}\right)=\frac{40}{63} \dot{n}_{X}^{\text {halo }} \frac{m_{\pi}^{3}-m_{\mu}^{3}}{m_{\pi}^{2}-m_{\mu}^{2}} \frac{1}{m_{\pi}}\left(\frac{m_{X}}{2}\right)^{1 / 2} E_{e}^{-3 / 2} .
$$


The ultra high energy electrons from pion decay are produced in the magnetic field of our galaxy, whose average strength will be denoted here by $B_{\mu}$ (in $\mu G$ ). The synchrotron emission of these electrons will typically result in the production of photons with energy

$$
E_{\gamma}=1.5 \cdot 10^{3} B_{\mu}\left(\frac{E_{e}}{10^{10} \mathrm{GeV}}\right)^{2} \mathrm{GeV} .
$$

Electrons with energy $E_{e} \geq 10^{17} \mathrm{eV}$ will then radiate photons with energy $E_{\gamma} \geq 0.1 \mathrm{GeV}$. Here we estimate the flux of these photons. The synchrotron emission is the dominant channel of energy losses at the energies of interest, and the time for losses is so small that all the electron energy is very rapidly radiated in the form of gamma rays, with flux

$$
I_{\gamma}\left(E_{\gamma}\right)=\frac{R_{H}}{4 \pi} q_{e}\left(E_{e}\right) \frac{E_{e}}{E_{\gamma}} \frac{d E_{e}}{d E_{\gamma}}=1.2 \cdot 10^{33}\left(\frac{m_{X}}{10^{14} G e V}\right)^{1 / 2} \dot{n}_{X}^{\text {halo }} B_{\mu}^{-1 / 4}\left(\frac{R_{H}}{100 k p c}\right) E_{\gamma}^{-7 / 4} \mathrm{~cm}^{-2} \mathrm{GeV}^{-1} \mathrm{~s}^{-1} \mathrm{sr}^{-1}
$$

where $\frac{d E_{e}}{d E_{\gamma}}$ has been derived from eq. (5). The normalized flux of gamma rays can now be calculated using eq. (3) which gives the following very interesting result

$$
I_{\gamma}\left(E_{\gamma}\right)=2.1 \cdot 10^{-9} B_{\mu}^{-1 / 4} E_{\gamma}^{-7 / 4} \mathrm{~cm}^{-2} \mathrm{GeV}^{-1} \mathrm{~s}^{-1} s r^{-1}
$$

independent on the size of the halo $R_{H}$ and the mass of the X-particles $m_{X}$.

The result is also weakly dependent on the average magnetic field $B_{\mu}$. For $B_{\mu} \sim 0.1-1 \mu G$ the flux derived from eq. (7) turns out to be detectable by EGRET in the energy range $100 \mathrm{MeV} \leq E_{\gamma} \leq$ few $\mathrm{GeV}$, and it is well above the sensitivity limit of Whipple for energies above $250 \mathrm{GeV}$.

The inverse dependence of $I_{\gamma}$ on $B_{\mu}$ can be easily explained on the basis of the electron spectrum resulting from the fragmentation function: such spectrum contains most of the energy at high electron energies. Therefore, at fixed $E_{\gamma}$ the larger electron energies (correspondent to smaller magnetic fields) contribute more, as far as the required electron energies are below $m_{X} / 2$.

The result quoted in eq. (7) changes appreciably as a function of the energy where $\mathrm{SH}$ relic particles provide the main contribution to the UHECR flux. This is more evident if we combine eqs. (2) and (6) to give: 


$$
I_{\gamma}\left(E_{\gamma}\right)=8.2 \cdot 10^{3} I_{\gamma}^{U H E}\left(E_{U H E}\right) E_{U H E}^{3 / 2} B_{\mu}^{-1 / 4} E_{\gamma}^{-7 / 4}
$$

where now $E_{U H E}$ is the energy at which we normalize the UHECR flux $I_{\gamma}^{U H E}$ (measured in units of $\left.\mathrm{cm}^{-2} \mathrm{~s}^{-1} \mathrm{sr}^{-1} \mathrm{GeV}^{-1}\right)$. From eq. (8) it results that if the normalization is carried out at $5 \times 10^{10} \mathrm{GeV}$, then the numerical factor in eq. (7) is $\sim 18$ times smaller. In this case, for $B_{\mu} \sim 1$ the flux drops below the detectability level of EGRET, though it remains still in the detectability range of next generation satellites (e.g. GLAST). In the region $E>100-200$ $\mathrm{GeV}$ the signal is comparable with the detectability levels of STACEE and WHIPPLE.

Let us come now to another interesting point: following the approach used in 14 and [12], the rate of decay of $\mathrm{SH}$ particles in the halo may be used to normalize the same quantity in the extragalactic space, through the relation

$$
\frac{\dot{n}_{X}^{\text {halo }}}{\dot{n}_{e g}}=\frac{\rho_{C D M}^{\text {halo }}}{\Omega_{C D M} \rho_{c r}}
$$

where $\dot{n}_{e g}$ is now the rate of $\mathrm{X}$ particle decay in the extragalactic space, $\rho_{C D M}^{\text {halo }}=0.3$ $\mathrm{GeV}_{\mathrm{cm}} \mathrm{m}^{-3}$ is the energy density of CDM in the halo, $\Omega_{C D M}$ is the fraction of CDM and $\rho_{c r}$ is the critical density of the Universe. We shall adopt here $\Omega_{C D M} h^{2}=0.2$. If we call $B_{\mu}^{e g}$ (in $\mu G$ ) the extragalactic magnetic field, the flux of gamma rays due to the synchrotron emission of UHEE can be calculated similarly to eq. (6) with $R_{H}$ substituted by $c t_{0}$, where $t_{0}=2.06 \cdot 10^{17} h^{-1} s$ is the age of the Universe and $h$ is the dimensionless Hubble constant. Of course this treatment is valid as far as gamma rays are not absorbed on scales much smaller than $c t_{0}$, which limits this estimate to $E_{\gamma}<500-1000 \mathrm{GeV}$. In this assumption the ratio of gamma rays with energy $E_{\gamma}$ produced in the extragalactic space relative to the ones produced in the halo is given by

$$
\frac{I_{\gamma}^{e g}\left(E_{\gamma}\right)}{I_{\gamma}\left(E_{\gamma}\right)}=0.23\left(\frac{R_{H}}{100 k p c}\right)^{-1}\left(\frac{\Omega_{C D M} h^{2}}{0.2}\right)\left(\frac{B_{\mu}}{B_{\mu}^{e g}}\right)^{1 / 4},
$$

where we used $h=0.6$. For extragalactic magnetic fields of order $10^{-9}-10^{-10}$ Gauss, and a typical galactic field of order of $\mu G$, the two gamma ray fluxes are comparable. Unfortunately only upper limits are currently available on the extragalactic magnetic field. Therefore in 
the following we shall not consider the extragalactic flux in any more detail and we shall concentrate our attention on the gamma ray flux from the halo.

\section{A DETAILED CALCULATION}

The simple calculations presented in the previous section suggest that the synchrotron emission of UHEE in the galactic magnetic field can produce the detectable signal in high energy gamma rays. The rate of production of electrons is normalized by requiring that the gamma rays generated in the decay of neutral pions saturate the flux of UHECR above some energy. As first pointed out in [6] and [12] the UHECR flux in this halo model is the generation spectrum resulting from the initial fragmentation process, hence it is dominated by gamma rays with respect to nucleons.

In this section we assume, following [12], that the rate of particle production is parametrized as the dark matter distribution [17]:

$$
\dot{n}_{X}^{h}(R)=\frac{\dot{n}_{0}^{h}}{\left(R / r_{0}\right)^{\gamma}\left[1+\left(R / r_{0}\right)^{\alpha}\right]^{(\beta-\gamma) / \alpha}}
$$

where $\dot{n}_{0}^{h}$ is the normalization rate, $R$ is the distance to the galactic center and $r_{0}$ is a

distance scale between 5 and $10 \mathrm{kpc}$. The parameters $\alpha, \beta, \gamma=(2,2,0)$ correspond to an isothermal profile, while $\alpha, \beta, \gamma=(2,3,0.2)$ give the best fit to observational data [17] and finally $\alpha, \beta, \gamma=(1,3,1)$ are obtained in the numerical simulation of ref. [18.

The calculation of the spectrum of hadrons produced in a single X-particle decay is given by the fragmentation function for the process $q \rightarrow$ hadrons. The fragmentation functions we use here are the LLA limiting spectrum of ordinary QCD [19] and its supersymmetric generalization given in [14] (hereafter SUSY-QCD). In the first case the fraction of pions produced in a single event is taken as $f_{\pi} \approx 1$, while in the second case we take, according with [14], $f_{\pi} \approx 0.5$. The pion fragmentation functions, indicated by $W_{\pi}\left(x, m_{x}\right)$ are then normalized as

$$
\int_{0}^{1} d x x W_{\pi}\left(x, m_{x}\right)=f_{\pi}
$$


where $x=2 E_{\pi} / m_{x}$. Approximately $1 / 3$ of $f_{\pi}$ is carried away by neutral pions while a fraction $2 / 3$ goes into charged pions. Each X-particle will produce two jets with energy $m_{X} / 2$ each.

Since the electrons radiate through synchrotron emission, a model for the galactic magnetic field is also needed. We parametrize the magnetic field in the form

$$
B(r, z)=B_{0}(r) \exp \left(-z / z_{0}\right)
$$

where $B_{0}(r)=3\left(r_{\odot} / r\right) \mu G$ when the radial distance $r$ in the disc is larger than $4 \mathrm{kpc}$ and $B_{0}(r)=$ const for $r<4 \mathrm{kpc}\left(\right.$ e.g. [20]). The parameter $z_{0}$ is fixed at $0.5 \mathrm{kpc}$ and $r_{\odot}=8.5 \mathrm{kpc}$ is the distance of the sun relative to the galactic center. $z$ is the height above the galactic disc.

According to [12 the flux of gamma rays with energy $E_{\gamma}$ produced in the $\pi^{0}$ decay is given by

$$
I_{\gamma}^{U H E}\left(E_{\gamma}\right)=\frac{W_{\gamma}\left(E_{\gamma}\right)}{4 \pi} \int_{-1}^{1} d \cos \theta|\cos \theta| \int_{0}^{r_{\max }(\theta)} d r \dot{n}_{X}^{h}(R)
$$

where $r_{\text {max }}(\theta)$ is easily calculated to be

$$
r_{\max }(\theta)=r_{\odot} \cos \theta+\sqrt{R_{h}^{2}-r_{\odot}^{2} \sin ^{2} \theta}
$$

and $R_{h}$ is the size of the halo. The angle $\theta$ is measured with respect to the direction of the galactic center. The gamma ray spectrum at the production is given by 12

$$
W_{\gamma}\left(E_{\gamma}\right)=\frac{4}{m_{X}} \int_{2 E_{\gamma} / m_{X}}^{1} \frac{d x}{x} W_{\pi^{0}}\left(x, m_{X}\right)
$$

with $W_{\pi^{0}}\left(x, m_{X}\right)=(1 / 3) W_{\pi}\left(x, m_{X}\right)$.

The comparison of the observed UHECR flux at $E=10^{10} \mathrm{GeV}$ with the flux of gamma rays from eqs. (14 16) at the same energy gives the normalizing value of $\dot{n}_{0}^{h}$ for each specific choice of the values of the parameters.

The spectrum of UHEE is the convolution of the pion and muon spectra and is given by

$$
W_{e}\left(E_{e}\right)=12 E_{e}^{2} \frac{m_{\pi}^{2}}{m_{\pi}^{2}-m_{\mu}^{2}} \frac{2}{m_{X}} \int_{E_{e}}^{m_{X} / 2} d E_{\mu} \frac{1}{E_{\mu}^{3}}\left[1-\frac{E_{e}}{E_{\mu}}\right] \int_{2 E_{\mu} / m_{X}}^{2 r^{2} E_{\mu} / m_{X}} \frac{d x}{x} W_{\pi^{ \pm}}\left(x, m_{X}\right),
$$


where $r=m_{\pi} / m_{\mu}$. The spectrum of electrons produced by a single muon is peaked at $E_{e} \approx(2 / 3) E_{\mu}$. In the simple assumption that all electrons are produced with this peak energy the above electron spectrum becomes

$$
W_{e}\left(E_{e}\right)=\frac{3}{m_{X}} \frac{m_{\pi}^{2}}{m_{\pi}^{2}-m_{\mu}^{2}} \int_{3 E_{e} / m_{X}}^{3 r^{2} E_{e} / m_{X}} \frac{d x}{x} W_{\pi^{ \pm}}\left(x, m_{X}\right) .
$$

As pointed out in the previous section the UHEE radiate by synchrotron emission in the galactic magnetic field in the gamma ray energy range, according with eq. (5). The emissivity per unit volume in the form of gamma rays with energy $E_{\gamma}$ at the position at distance $R$ from the galactic center can be written as

$$
q_{\gamma}\left(E_{\gamma}, r, z\right)=\dot{n}_{X}^{h}(R) W_{e}\left(E_{e}\right) \frac{E_{e}}{E_{\gamma}} \frac{d E_{e}}{d E_{\gamma}}
$$

where $E_{e}$ and $E_{\gamma}$ are related through eq. (5) but the magnetic field is now a function of the position in the galaxy. Hence the same $E_{\gamma}$ will correspond to different $E_{e}$ in different positions in the galaxy.

Since the magnetic field is symmetric with respect to the disc while the dark matter profile is spherically symmetric, the integration over lines of sight requires the explicit integration over both $\theta$ and $\phi$, so that the flux of gamma rays at the Earth, per unit time, surface, energy and solid angle is

$$
I_{\gamma}\left(E_{\gamma}\right)=\frac{1}{4 \pi} \int_{0}^{2 \pi} d \phi \int_{-1}^{1} d \cos \theta|\cos \theta| \int_{0}^{r_{\max }(\theta)} d r q_{\gamma}\left(E_{\gamma}, r, z\right),
$$

where $r_{\max }(\theta)$ is again given by eq. (15).

The results of the calculations are plotted in Figs. 1 and 2, where we used $r_{0}=10 \mathrm{kpc}$ and $z_{0}=0.5 \mathrm{kpc}$. Although the calculation has been carried out for the three sets of values of the $\alpha, \beta, \gamma$ parameters, the results are quite insensitive to the specific model of the halo distribution of dark matter, and the related curves would be indistiguishable. Therefore we plotted there only the case $(\alpha, \beta, \gamma)=(2,2,0)$ for reference. In Fig. 1 we plotted $E_{\gamma}^{1.75} I_{\gamma}\left(E_{\gamma}\right)$ in order to amplify the spectral differences. The two thick lines refer to $m_{X}=10^{14} \mathrm{GeV}$, while the two thin lines are for $m_{X}=10^{13} \mathrm{GeV}$. The solid curves are obtained using the 
SUSY-QCD fragmentation function while the dashed lines are calculated with the ordinary QCD fragmentation function. For comparison we also plotted by a dash-dotted line the extragalactic diffuse gamma ray background [28]. All our fluxes are below this limit in the energy range accessible to current experiments. The points with arrows are the upper limits on the gamma ray flux from the HEGRA [21], Utah-Michigan [22], EAS-TOP [23] and CASA-MIA 24 experiments.

Clearly the stronger constraints on the gamma ray flux from the halo are imposed by the CASA-MIA and EAS-TOP experiments. In most of the cases considered in this paper the fluxes are below these limits, with the exception of the case $m_{X}=10^{14} \mathrm{GeV}$ with QCD fragmentation function (thick dashed line), though this is a small effect and not enough to rule out this case.

In Fig. 2 the gamma ray fluxes have been blown up in the energy range between 100 $\mathrm{MeV}$ and $10^{4} \mathrm{GeV}$ (lines labelled as before) and compared with the sensitivity of some of the present or planned gamma ray experiments operating or planned in the same energy range (e.g. EGRET, GLAST, STACEE and WHIPPLE). The comparison confirms that the signal can be detected. It could be particularly interesting to look at the gamma ray signal in the region above $\sim 500 \mathrm{GeV}$, where the contamination due to the isotropic diffuse gamma ray background, believed to be of extragalactic origin, should be reduced due to absorption of gamma rays on the infrared background. Our calculations show an integrated flux above $500 \mathrm{GeV}$ at the level of $\sim 10^{-11}$ photons $\mathrm{cm}^{-2} \mathrm{~s}^{-1} \mathrm{sr}^{-1}$, well above the detectability limit of the Whipple experiment.

The statement, based on eq. (7), that the gamma ray flux is not dependent on $m_{X}$ and $R_{H}$ is confirmed here. There is clearly a weak dependence on $m_{X}$ introduced by the fragmentation functions, more complicated than the simple power law adopted in section [11. The difference is particularly evident on the tail of the spectra where the highest energy electrons (with energy close to $\sim m_{X} / 2$ ) are probed. We carried out the calculations also with $m_{X}=10^{12} \mathrm{GeV}$ : the gamma ray spectra fall down appreciably already at $E_{\gamma} \sim 10^{4}$ $\mathrm{GeV}$ and the flux at $E_{\gamma} \sim 100 \mathrm{MeV}$ is a factor $\sim 2$ larger than in the other cases. 
We also checked that our predictions do not depend appreciably on the typical scale height $z_{0}$ of the magnetic field (eq. (13)).

The energy of UHECR at which we normalize the decay rate of SH particles per unit volume turns out to be a critical parameter: the fluxes in Figs. 1-2 decrease by approximately one order of magnitude if $\mathrm{SH}$ relic particles provide the main contribution to the UHECR spectrum at $\sim 5 \cdot 10^{10} \mathrm{GeV}$ instead of $10^{10} \mathrm{GeV}$. In this case the gamma ray fluxes are much closer to the detectability limits of WHIPPLE and STACEE in the range $100-10^{4} \mathrm{GeV}$ and below the EGRET sensitivity at $\mathrm{GeV}$ energies (see also eq. (8)), though the fluxes remain above the detection limit of GLAST.

In this sense the fluxes quoted above can be considered as upper limits on the gamma ray emission from $\mathrm{SH}$ particles in the halo.

\section{CONCLUSIONS}

We calculated the flux of gamma radiation produced through synchrotron emission in the galactic magnetic field from ultra-high energy electrons generated as decay product of charged pions in the hadronic cascade coming from the decay of X-particles, when they cluster in the galactic halo in the form of $\mathrm{SH}\left(m_{X} \sim 10^{13}-10^{14} \mathrm{GeV}\right)$ quasistable particles.

If the magnetic field in the galactic halo is $B_{\text {halo }} \sim 0.1-1 \mu G$, UHEE with energy between $10^{17} \mathrm{eV}$ and $\sim m_{X} / 2$ very rapidly loose all their energy in the form of gamma rays mostly with energies $E_{\gamma} \leq 10^{6}-10^{8} \mathrm{GeV}$. The resulting diffuse gamma ray emission above $100 \mathrm{MeV}$ is of the order of $10^{-8}$ photons $\mathrm{cm}^{-2} \mathrm{~s}^{-1} \mathrm{sr}^{-1}$ (Fig. 1). This result is not appreciably dependent on the mass $m_{X}$ of the SH particles nor on the geometry of the galactic dark matter halo, but depends critically on the energy where the contribution of $\mathrm{SH}$ relic particles to UHECR becomes dominant (section III): the fluxes decrease by a factor $\sim 18$ if the normalization to the UHECR flux is done at $5 \cdot 10^{10} \mathrm{GeV}$ instead of $10^{10} \mathrm{GeV}$.

In section $\mathbb{1}$ we also estimated the extragalactic flux of gamma rays due to the same physical process, and found that, for typical extragalactic magnetic fields of $\sim 10^{-10}-10^{-9}$ 
Gauss the extragalactic and the halo fluxes are comparable, if SH particles provide a fraction $\Omega_{C D M} h^{2}=0.2$ of the CDM and the SH particles in the halo explain the UEHCR flux (the galactic and extragalactic densities of SH particles are related through eq. (9)).

It is interesting to note that the detection of a residual gamma ray emission from the halo, after the subtraction of the contribution of $p p$ collisions, Inverse Compton scattering, electron bremsstrahlung and of the extragalactic diffuse gamma ray background was recently claimed. This subtraction procedure is quite difficult and very model dependent; however the analysis in [25] and [26] show that the residual emission is at the level of $\sim 10^{-7}$ photons $c m^{-2} s^{-1} s r^{-1}$ above $1 \mathrm{GeV}$ with a spectrum $E_{\gamma}^{-\alpha}$ and $\alpha \approx 1.8$. This flux, if confirmed, is a factor $\sim 10$ larger than what is found in the present calculation with the normalization at the UHECR flux carried out at $10^{10} \mathrm{GeV}$, and the power index $\alpha \approx 1.8$ is close to 1.75 in eq. (7). In the more realistic calculations illustrated in section [1] the spectrum cannot be approximated by a unique power law but in the energy region between $100 \mathrm{MeV}$ and a few $\mathrm{GeV}$ the slope of the spectra in the log-log plot is around 1.8 - 1.9.

The origin of the residual emission is still under debate and the possibility of an underestimate of the contribution of inverse Compton scattering is an open possibility, so that the actual residual emission (if any) could be smaller than what previously claimed. In this prospective it is interesting to study the contribution of $\mathrm{SH}$ particles in the halo to this emission as an open intriguing possibility requiring further investigation, though probably only next generation gamma ray detectors can shed a new light on the problem.

A particularly interesting aspect is the anisotropy of the gamma ray emission expected in this model [27]. The anisotropy is the result of both the asymmetric configuration of the magnetic field in the galaxy as seen from the Earth, and the asymmetric position of the solar system in the galaxy (this also produces some anisotropy in the arrival directions of UHECR [11]13]).

The upper limits reported in Fig. 1 are due to the HEGRA [21], Utah-Michigan [22], EASTOP [23] and CASA-MIA [24] experiments. The stronger constraints to the model of SH particles in the halo come from the CASA-MIA and EASTOP experiments, though all 
the calculated fluxes are compatible with the experimental limits (only two data points from the CASA-MIA experiment fall slightly below the tail of the curve obtained for $m_{X}=10^{14}$ $\mathrm{GeV}$ with the QCD fragmentation function).

In Fig. 2 it is particularly clear that the predicted fluxes of gamma rays are well above the detectability limits of the STACEE (for $E_{\gamma} \geq 20 \mathrm{GeV}$ ) and WHIPPLE (for $E_{\gamma} \geq 250$ $\mathrm{GeV}$ ) experiments when the $\mathrm{SH}$ relic particles dominate the UHECR flux at $10^{10} \mathrm{GeV}$.

We propose that it could be of particular interest to study the gamma ray emission from the halo in the energy range $E_{\gamma} \geq 500-1000 \mathrm{GeV}$, for two reasons: a) the diffuse extragalactic gamma ray background is expected to be less pronounced in this energy range because of absorption of gamma rays in the intergalactic medium on the infrared photon

background; b) the spectrum of the gamma radiation from $\mathrm{SH}$ relic particles is predicted to have a spectrum $E_{\gamma}^{-\alpha}$ with $\alpha \approx 1.7-2$, substantially flatter than the gamma ray emission from $p p$ collisions (expected to have the same spectrum as the cosmic rays) which should dominate over other mechanisms at these high energies.

\section{Aknowledgments}

The author is grateful to V.S. Berezinsky and A. Olinto for many useful discussions that helped understanding the proposed effect and for a critical reading of the manuscript. The research of P.B. is funded by INFN at the University of Chicago. 


\section{Figure Captions}

Fig. 1: Flux of gamma rays from synchrotron emission of UHEE in the galactic magnetic field for $m_{X}=10^{14} \mathrm{GeV}$ (thick lines) and $m_{X}=10^{13} \mathrm{GeV}$ (thin lines). The solid lines are obtained with the SUSY-QCD fragmentation function [14, while the dashed lines are for the ordinary QCD fragmentation function [19]. Fluxes are multiplied by $E_{\gamma}^{1.75}$ to emphasize the spectral differences among the curves. Upper limits are from the HEGRA [21], UtahMichigan [22], EASTOP [23] and CASA-MIA [24] experiments. The dash dotted line is the extragalactic diffuse gamma ray background from EGRET [28].

Fig. 2: Flux of gamma rays (multiplied by $E_{\gamma}$ ) for the same cases as in Fig. 1 but

limited to the energy range $100 \mathrm{MeV} \leq E_{\gamma} \leq 10^{4} \mathrm{GeV}$. Lines are labelled as in Fig. 1. The thresholds for detectability of the EGRET, GLAST, STACEE and WHIPPLE experiments are drawn. 


\section{REFERENCES}

[1] M. Takeda et al., preprint astro-ph/9807193.

[2] K. Greisen, Phys. Rev. Lett. 16, 748 (1966); G.T. Zatsepin and V.A. Kuzmin, Sov. Phys. JETP Lett. 4, 78 (1966).

[3] P.P. Kronberg, Rep. Prog. Phys. 57, 325 (1994).

[4] P. Blasi, S. Burles and A. Olinto, preprint astro-ph/9812487, accepted for publication in Astroph. J. Lett.

[5] P. Bhattacharjee and G. Sigl, preprint astro-ph/9811011 (submitted to Phys. Rep.).

[6] V.S. Berezinsky, M. Kachelriess and A. Vilenkin, Phys. Rev. Lett. 79, 4302 (1997).

[7] M. Birkel and S. Sarkar, Astrop. Phys. 9, 297 (1998).

[8] V.A. Kuzmin, at the Workshop Beyond the Desert, Castle Ringberg, June 1997 (preprint astro-ph/9709187) and International Workshop on Non Accelerator New Physics, Dubna, july 1997.

[9] V.S. Berezinsky, preprint astro-ph/9811268.

[10] D.J.H. Chung, E.W. Kolb and A. Riotto, preprint astro-ph/9805473; D.J.H. Chung, E.W. Kolb and A. Riotto, preprint astro-ph/9809453.

[11] S.L. Dubovsky and P.G. Tinyakov, preprint hep-ph/9802382.

[12] V.S. Berezinsky, P. Blasi and A. Vilenkin, Phys. Rev. D58, 103515 (1998).

[13] V.S. Berezinsky and A. Mikhailov, preprint astro-ph/9810277.

[14] V.S. Berezinsky and M. Kachelriess, Phys. Lett. B434, 61 (1998).

[15] C.T. Hill, Nucl. Phys. B224, 469 (1983).

[16] C.T. Hill, D.N. Schramm and T.P. Walker, Phys. Rev. D36, 1007 (1987). 
[17] A.V. Kravtsov, A.K. Klypin, J.S. Bullock and J.R. Primack, preprint astro-ph/9708176.

[18] J.F. Navarro, C.S. Frenk and S.D.M. White, Astroph. J. 462, 563 (1996).

[19] Yu.L. Dokshitzer, V.A. Khose, A.H. Mueller and S.I. Troyan, Basics of Perturbative QCD (Editions Frontières, Gif-sur-Yvette, France, 1991).

[20] T. Stanev, Astroph. J. 479, 290 (1997).

[21] A. Karle et al., Phys. Lett. B347, 161 (1995).

[22] J. Matthews et al., Astroph. J. 375, 202 (1991).

[23] M. Aglietta et al., Astrop. Phys. 6, 71 (1996).

[24] M.C. Chantell et al., Phys. Rev. Lett. 79, 1805 (1997).

[25] D.D. Dixon et al., New Astronomy 3, 539 (1998).

[26] R. Chary and E.L. Wright, preprint astro-ph/9811324.

[27] P. Blasi and A. Olinto, in preparation.

[28] A. Chen, J. Dwyer and P. Kaaret, Astroph. J. 463, 169 (1996); P. Sreekumar et al., Astroph. J. 494, 523 (1998). 


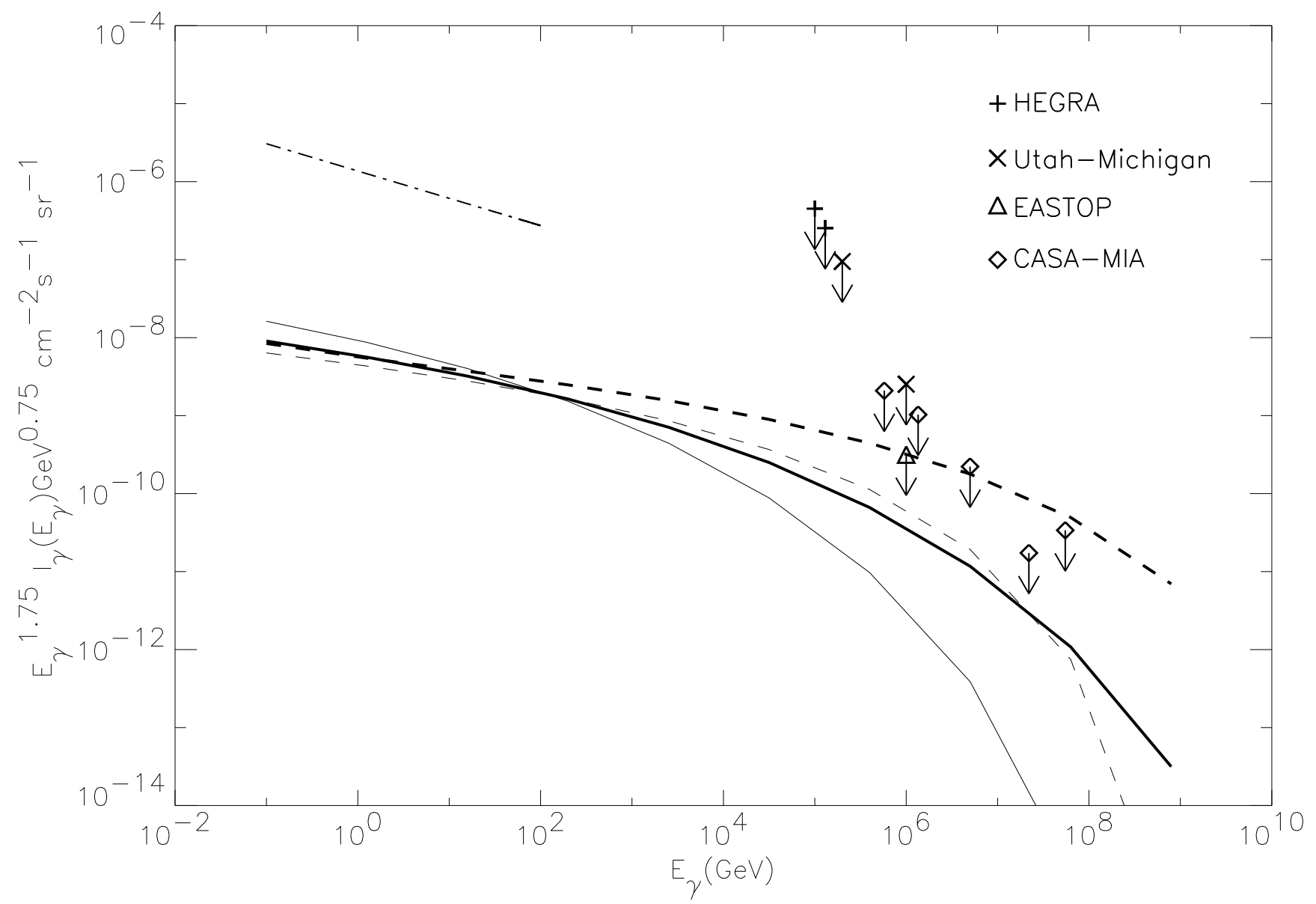




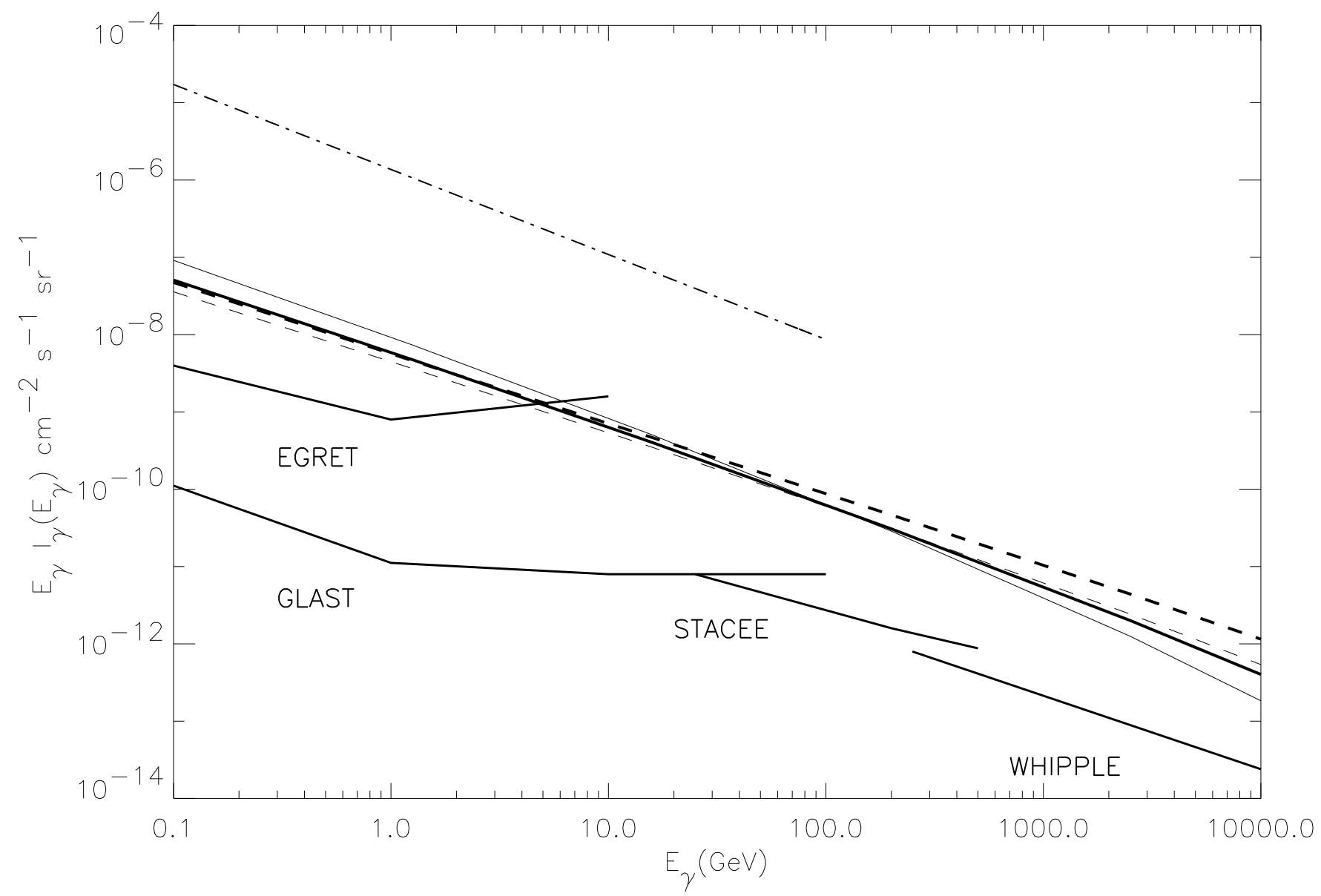

